\title{
Relative nutritional deficiencies associated with centrally acting monoamines
}

Marty Hinz'

Alvin Stein ${ }^{2}$

Thomas Uncini ${ }^{3}$

'Clinical Research, NeuroResearch Clinics Inc, Cape Coral, ${ }^{2}$ Stein

Orthopedic Associates, Plantation, FL,

${ }^{3}$ DBS Labs, Duluth, MN, USA
Correspondence: Marty Hinz 1008 Dolphin Drive, Cape Coral, FL 33904, USA

Tel + I 2186262220

Fax + I 2186261638

Email marty@hinzmd.com
This article was published in the following Dove Press journal:

International Journal of General Medicine

7 May 2012

Number of times this article has been viewed

Background: Two primary categories of nutritional deficiency exist. An absolute nutritional deficiency occurs when nutrient intake is not sufficient to meet the normal needs of the system, and a relative nutritional deficiency exists when nutrient intake and systemic levels of nutrients are normal, while a change occurs in the system that induces a nutrient intake requirement that cannot be supplied from diet alone. The purpose of this paper is to demonstrate that the primary component of chronic centrally acting monoamine (serotonin, dopamine, norepinephrine, and epinephrine) disease is a relative nutritional deficiency induced by postsynaptic neuron damage.

Materials and methods: Monoamine transporter optimization results were investigated, reevaluated, and correlated with previous publications by the authors under the relative nutritional deficiency hypothesis. Most of those previous publications did not discuss the concept of a relative nutritional deficiency. It is the purpose of this paper to redefine the etiology expressed in these previous writings into the realm of relative nutritional deficiency, as demonstrated by monoamine transporter optimization. The novel and broad range of amino acid precursor dosing values required to address centrally acting monoamine relative nutritional deficiency properly is also discussed.

Results: Four primary etiologies are described for postsynaptic neuron damage leading to a centrally acting monoamine relative nutritional deficiency, all of which require monoamine transporter optimization to define the proper amino acid dosing values of serotonin and dopamine precursors.

Conclusion: Humans suffering from chronic centrally acting monoamine-related disease are not suffering from a drug deficiency; they are suffering from a relative nutritional deficiency involving serotonin and dopamine amino acid precursors. Whenever low or inadequate levels of monoamine neurotransmitters exist, a relative nutritional deficiency is present. These precursors must be administered simultaneously under the guidance of monoamine transporter optimization in order to achieve optimal relative nutritional deficiency management. Improper administration of these precursors can exacerbate and/or facilitate new onset of centrally acting monoamine-related relative nutritional deficiencies.

Keywords: nutritional deficiency, serotonin, dopamine, monoamine

\section{Introduction}

It is much more desirable to identify, address, and eliminate the cause of a disease than to treat its symptoms. Until this research project defined the relative nutritional deficiencies associated with disease and dysfunction of the centrally acting monoamines due to low or inadequate levels of neurotransmitters, there was no awareness of these nutritional deficiencies and no ability to address them properly and optimally. ${ }^{1}$ The authors of this paper have published extensively on the topic of monoamine amino acid precursor 
management relating to various diseases and dysfunctions. Further research in the areas covered in the previous writings has revealed a relative nutritional deficiency (RND) etiology not previously recognized or reported. The novel concept of a monoamine-related RND is developed in this paper. ${ }^{1-13}$

Serotonin, dopamine, norepinephrine, and epinephrine are "centrally acting monoamines" (herein referred to as monoamine[s]), and are also involved in the control and regulation of peripheral functions.

This novel concept hypothesizes the etiology of chronic disease and/or regulatory dysfunctional symptoms to be inadequate levels of monoamines as opposed to low levels of synaptic monoamines. The RND described herein are the most prevalent type of nutritional deficiency afflicting humans. An extensive list of diseases, conditions, and dysfunctions has been identified in which synaptic monoamine RND are recognized (see Appendix A and Appendix B). ${ }^{1-13}$ It is postulated that over $80 \%$ of humans suffer from symptoms relating to a serotonin and/or catecholamine RND. Monoamine-related RND was unrecognized prior to this research due to the inability to manage and verify results of monoamine transporter manipulation objectively. The organic cation transporters (OCT) are the primary determinants of intercellular and extracellular monoamine concentrations throughout the body.

\section{Absolute nutritional deficiency versus RND}

Two primary categories of nutritional deficiency exist, ie, absolute nutritional deficiency and RND. ${ }^{1}$ Insufficient dietary nutrient intake causes absolute nutritional deficiencies. An absolute nutritional deficiency can be corrected by optimizing nutrient intake in the diet. Management of the problem is often enhanced by administration of nutritional supplements, but they are not required. ${ }^{1}$

When an RND exists, nutritional intake and systemic nutrient levels are normal. However, systemic needs are increased above normal by outside forces and cannot be achieved by dietary modification alone. Burns and postsurgical patients are examples where an RND may develop. ${ }^{1}$

In this paper, the authors discuss the novel finding that an RND is the primary etiology whenever there is a chronic disease or dysfunction relating to a compromise in the flow of electricity through the presynaptic neurons (axons) across the synapses then through the postsynaptic neurons (dendrites). An extensive list of diseases, conditions, and dysfunctions has been identified in which synaptic monoamine RND are recognized (see Appendix A and Appendix B). ${ }^{1-13}$
The monoamine-associated RND is by far the most prevalent constellation of nutritional deficiencies found in humans (see Appendix A and Appendix B). It is postulated that over $80 \%$ of humans suffer from symptoms relating to a serotonin and/or catecholamine RND. Conditions prior to in situ monoamine transporter optimization (MTO, referred to in some previous papers as OCT functional status optimization) made it impossible to achieve consistent results with the administration of monoamine amino acid precursors. With the invention and refinement of MTO, the ability to study, manipulate, and optimally manage monoamine-related RND became clinically possible. ${ }^{1-13}$

Four primary classes of monoamine-associated RND have been identified by this research project:

- RND associated with monoamine disease or dysfunction

- RND induced by inappropriate administration of amino acids

- RND induced iatrogenically or by the administration of certain drug classes

- RND associated with genetic defects or predisposition.

\section{Endogenous versus competitive inhibition state}

Serotonin and dopamine, and their precursors, exist in one of two distinctly unique and physiologically divergent states, ie, the endogenous state and the competitive inhibition state. $^{1-8,11-13}$ The monoamine hypothesis advocates that the etiology of disease symptoms and/or regulatory dysfunction in the endogenous state is low synaptic monoamine concentrations which induce trans-synaptic electrical defects. Under this model, an absolute nutritional deficiency type of approach is advocated, where simply returning synaptic monoamine levels to normal corrects the electrical problem, leading to relief of disease symptoms. None of this is true. There is no documentation illustrating that merely establishing normal synaptic neurotransmitter levels is effective in correcting an electrical defect. ${ }^{1}$

Subjects in the endogenous state, with and without monoamine-related disease, if not suffering from a monoamine-secreting tumor, cannot be differentiated by laboratory monoamine assays including MTO. Statistical distribution of monoamine levels are the same in subjects with and without disease. ${ }^{1,5,7,11}$

The term "competitive inhibition" refers primarily to interaction between monoamines and their amino acid precursors in synthesis, metabolism, and transport. The competitive inhibition state occurs when significant amounts of serotonin and dopamine amino acid precursors are administered simultaneously. 
The daily dosing values of serotonin and/or dopamine precursors required for the system to enter into the competitive inhibition state cannot be achieved by diet alone. When the etiology of chronic symptoms is postsynaptic electrical compromise, the system needs to be placed into the competitive inhibition state in order to elevate synaptic monoamines high enough to compensate for the postsynaptic damage. Optimization of synaptic monoamine levels in order to facilitate optimal flow of electricity is only possible with simultaneous administration of serotonin and dopamine precursors guided by MTO. ${ }^{1-8,11-13}$

\section{Etiologies of postsynaptic neuron damage}

Significant damage to the postsynaptic neurons of the serotonin and catecholamine systems may theoretically have numerous etiologies. The most common ${ }^{1}$ are (in order of frequency of occurrence):

- neurotoxin-induced

- trauma-related

- biology-related

- genetic predisposition.

These four categories interact and are interconnected. For example, patients suffering from the genetic disease CharcotMarie-Tooth, which afflicts approximately 1 in 2500 humans, have a list of over 50 drugs that are potentially neurotoxic in the presence of this genetic state but are not toxic to patients without the genetic disorder. ${ }^{14}$

In patients suffering from chronic monoamine-related disease, there is permanent damage to the structures of the postsynaptic neurons that conduct electricity, such as occurs in Parkinson's disease. ${ }^{1}$ This damage is permanent and does not spontaneously reverse with time. Parkinson's disease is not the only monoamine-related disease where humans suffer significant permanent postsynaptic damage. ${ }^{6}$ Based on MTO results, virtually all patients with chronic monoamine-associated disease have permanent postsynaptic damage caused by outside forces. ${ }^{1}$

\section{Synaptic monoamine levels}

These monoamines do not cross the blood-brain barrier. Drugs do not increase the total number of monoamine molecules in the brain; their mechanism of action only facilitates movement of monoamines from one place to another. The only way to increase the total number of monoamine molecules in the brain is by administration of their amino acid precursors which cross the blood-brain barrier where they are then synthesized into new monoamine molecules. ${ }^{1}$

Serotonin is synthesized from 5-hydroxytryptophan (5-HTP) which is synthesized from L-tryptophan. Dopamine is synthesized from L-dopa which is synthesized from L-tyrosine. Epinephrine is synthesized from norepinephrine which is synthesized from dopamine (see Figure 1). ${ }^{1,8}$

Prior to development of MTO, no method existed to manage properly and objectively the amino acid and monoamine interaction problems found in Figure 2 that are observed in the competitive inhibition state. The very act of administering amino acid precursors may cause amino acid and/or monoamine depletion, leading to an RND. The administration of improperly balanced amino acids may lead to an RND environment with increased side effects, adverse reactions, and suboptimal results. ${ }^{1,8}$

The key to addressing an amino acid precursor imbalance during administration is the novel method of simultaneous administration of serotonin and dopamine precursors, along with sulfur amino acids in a proper balance, as defined by MTO. ${ }^{1,8}$

Review of the chemical properties of the immediate monoamine precursors, L-dopa and 5-HTP, shows that they hold tremendous and extraordinary potential in the management of RND. L-dopa and 5-HTP are freely synthesized to dopamine and serotonin, respectively, without biochemical feedback inhibition. Each freely crosses the blood-brain barrier. It is possible to achieve any required level of serotonin and dopamine to optimize synaptic monoamine levels in the brain with these nutrients. MTO reveals that it is not the concentration of monoamines that is critical for optimal results; it is the balance between serotonin and dopamine in the competitive inhibition state, as defined by MTO, that is most critical in re-establishing and optimizing the postsynaptic flow of electricity. ${ }^{1,8}$

Even though 5-HTP has had increasing usage by physicians, the literature, dating back to the 1950s, has never

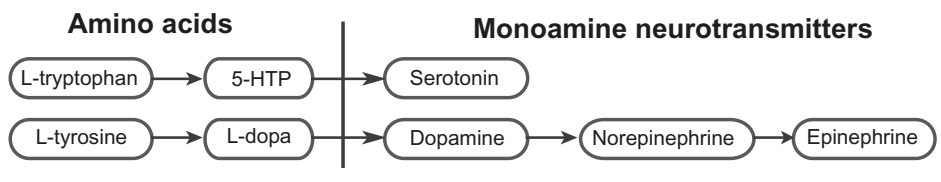

Figure I Synthesis of serotonin and the catecholamines (dopamine, norepinephrine, and epinephrine). Abbreviations: 5-HTP, 5-hydroxytryptophan; L-dopa, L-3,4-dihydroxyphenylalanine. 


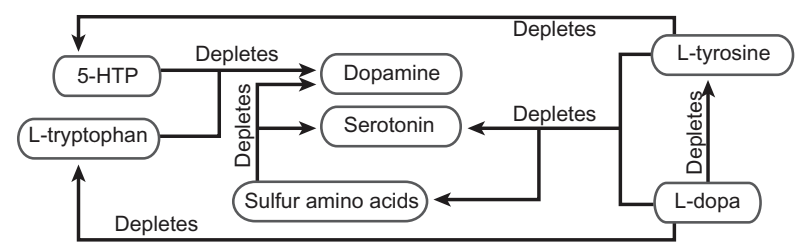

Figure 2 Amino acid precursor-induced monoamine relative nutritional deficiency. Administration of improperly balanced monoamine precursors and/or sulfur amino acids may lead to far reaching relative nutritional deficiencies. This depletion of amino acids and monoamines can only be corrected with proper administration of nutrients as guided by monoamine transporter optimization.

Abbreviations: 5-HTP, 5-hydroxytryptophan; L-dopa, L-3,4-dihydroxyphenylalanine.

supported truly successful, consistent, and reproducible use of 5-HTP in management of nutritional deficiencies. MTO clearly explains this problem, ie, the unbalanced approach to the use of amino acid precursors. ${ }^{8}$

The literature on Parkinson's disease demonstrates that the L-dopa dosing value potential is limited due to side effects and adverse reactions. In addition, the effectiveness of L-dopa wanes with time (tachyphylaxis). While L-dopa is recognized as the most effective nutritional management for Parkinson's disease, it is common to use other much less effective alternative Parkinson's medications, such as agonists and metabolic enzyme inhibitors, initially and for as long as possible in the management of Parkinson's disease, saving L-dopa for last due to all of the problems and side effects associated with its unbalanced administration. As noted in previous writings, utilizing MTO technology will virtually eliminate all side effects and problems associated with L-dopa administration which stem from improper balance of serotonin, 5-HTP, L-tryptophan, L-tyrosine, and sulfur amino acids.

Optimal 5-HTP and L-dopa results require MTO. It is only under these conditions that efficacy increases significantly, and side effects virtually resolve or are made manageable. ${ }^{6}$ Specific examples of the dominant monoamine depleting the nondominant monoamine are listed here and illustrated in Figure 2.,8

- 5-HTP may deplete dopamine

- L-tryptophan may deplete dopamine

- L-dopa may deplete serotonin

- L-dopa may deplete L-tryptophan

- L-dopa may deplete L-tyrosine

- L-dopa may deplete sulfur amino acids

- L-tyrosine may deplete serotonin

- L-tyrosine may deplete 5-HTP

- L-tyrosine may deplete sulfur amino acids

- Sulfur amino acids may deplete dopamine
- Sulfur amino acids may deplete serotonin

MTO in the competitive inhibition state reveals that effecting change to one component will effect change to all components of the serotonin-catecholamine system, as depicted in Figure 2, in a predictable manner. Unbalanced administration of precursors causes the dominant monoamine to exclude the nondominant monoamine in synthesis and transport, leading to depletion and evolution of an RND relating to the nondominant system in the process. A novel finding of this research is that when depletion of the nondominant system is great enough, the effects of the dominant system will no longer be observed at any dosing value. This is a severe RND state. ${ }^{8}$

The L-aromatic amino acid decarboxylase enzyme catalyzes conversion of 5-HTP and L-dopa to serotonin and dopamine, respectively. Reviewing Figure 3, administration of unbalanced enzyme-dominant dosing values of 5-HTP or L-tryptophan will cause the serotonin side of the equation to dominate L-aromatic amino acid decarboxylase and deplete the dopamine/catecholamine side of the equation through compromise of synthesis. This causes an RND of the nondominant dopamine/catecholamine systems. The same is true in reverse with the administration of L-dopa. When the dopamine side is dominant at the enzyme relative to the serotonin side, a serotonin-related RND will occur (see Figures 2 and 3). ${ }^{1-8,11-13}$

The activity of the monoamine oxidase enzyme system, which catalyzes monoamine metabolism, is not static. If levels of one system become dominant, monoamine oxidase activity will increase, leading to depletion and an associated RND of the nondominant system via accelerated metabolism (see Figures 2 and 4). ${ }^{1-8,11-13}$

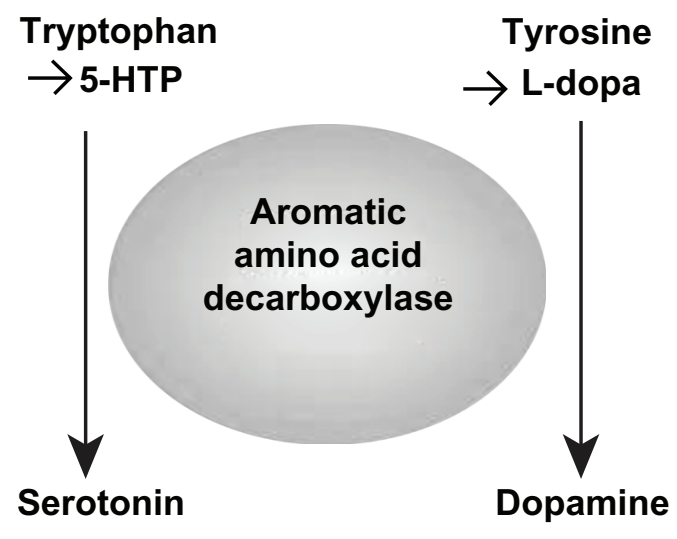

Figure 3 Improperly balanced amino acid precursor administration leads to depletion of the nondominant system causing a relative nutritional deficiency of that system through competitive inhibition at the L-aromatic amino acid decarboxylase by the dominant system during synthesis of serotonin and dopamine. Abbreviations: 5-HTP, 5-hydroxytryptophan; L-dopa, L-3,4-dihydroxyphenylalanine. 


\section{Dopamine norepinephrine epinephrine}

\section{Serotonin}

$\downarrow_{\text {HVA }}$

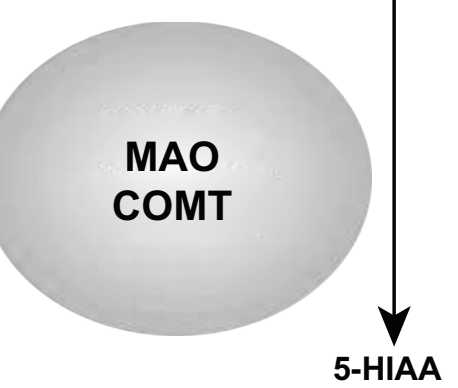

Figure 4 Domination of the monoamine oxidase enzyme system by one system leads to increased enzyme activity resulting in depletion of the nondominant system with an associated relative nutritional deficiency through increased metabolism. Abbreviations: COMT, catechol-O-methyltransferase; MAO, monoamine oxidase; 5-HIAA, 5-hydroxyindoleacetic acid; HVA, homovanillic acid.

Synthesis (Figure 3) and metabolism (Figure 4) of monoamines is dependent on OCT which regulates movement of amino acids and monoamines in and out of cellular structures where these functions take place. This functional status can only be determined in situ with monoamine oxidase..$^{1-8,11-13}$

OCT-dependent metabolism takes place both inside and outside of cells. If one system dominates the transporter, the nondominant system will be excluded from transport, leading to suboptimal regulation of function secondary to increased metabolism, and decreased synthesis of the nondominant system (see Figure 5). When unbalanced amino acid precursors and/or monoamines are present at the transporter entrance, systemic monoamine concentrations, which are dependent on transport, will not be optimal. This leads to an amino acid-induced RND along with suboptimal regulation of function..$^{1-8,11-13}$

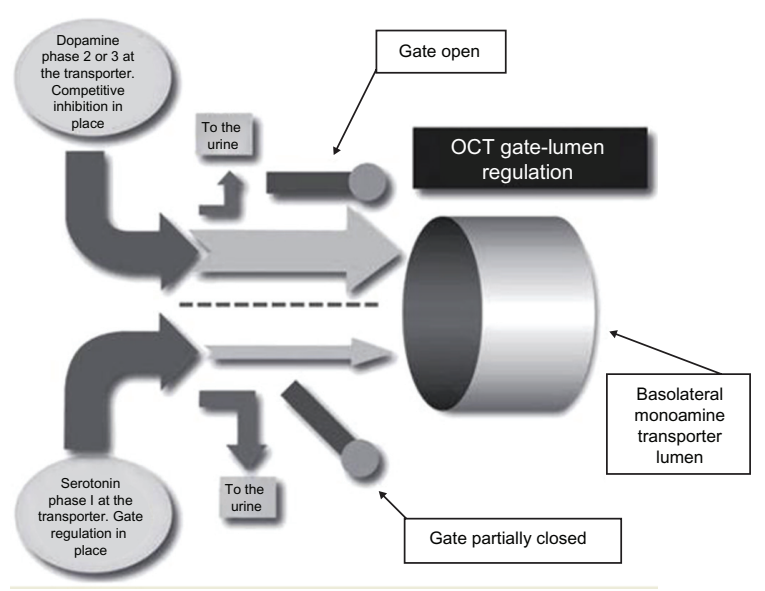

Figure $\mathbf{5}$ In the competitive inhibition state, organic cation transport of serotonin and catecholamines needs to be in proper balance to ensure optimal regulation of function and optimal synthesis of both systems and prevent monoamine-induced and/or amino acid-induced relative nutritional deficiencies.

Abbreviation: OCT, organic cation transporters.
When the established effects of the dominant system dissipate, secondary to depletion of the nondominant system, it is caused by an amino acid-induced RND associated with the nondominant monoamine system. This research has tracked the etiology of L-dopa tachyphylaxis to a novel serotonin-related $\mathrm{RND}$, ie, serotonin is depleted due to serotonin precursor nutrient needs being greater than can be achieved with an optimal diet in the face of L-dopa depletion of serotonin and serotonin precursors. This is supported by the novel findings that administering proper levels of serotonin precursors as guided by MTO can reverse L-dopa tachyphylaxis quickly. ${ }^{1-8}$

\section{Centrally acting monoamine RND}

The bundle damage theory notes that damage to the postsynaptic structural components involved with electrical conduction is the primary cause of electrical dysfunction associated with the monoamine-related diseases, not low synaptic neurotransmitter levels. As previously noted, when these electrical dysfunctions are present on a chronic basis, monoamine levels and nutrient levels are in the normal range on laboratory studies. ${ }^{1,8}$ The damage to the postsynaptic neurons leads to a compromise in the regulatory flow of electricity. When the flow of electricity is compromised enough, symptoms and dysfunction develop. ${ }^{1}$

Parkinson's disease is a prototype in the study of monoamine-related RND. It is well known that in Parkinson's disease there is damage to the dopamine neurons of the substantia nigra in the brain. L-dopa is administered in order to increase dopamine levels to compensate for the compromised electrical flow that results from the damage.

MTO evaluation shows that the only viable explanation for chronic electrical dysfunctional diseases that are present in patients who have normal synaptic monoamine levels is damage to the postsynaptic neuron structures (bundle damage theory). This is the classical presentation observed with the Parkinson's disease model where electrical dysfunction secondary to postsynaptic neuron damage has been identified and has caused an RND problem related to inadequate intake of the dopamine precursor. It is the novel findings of this research project that, as with Parkinson's disease, postsynaptic neuronal damage with the associated RND is common in all chronic monoamine-related illnesses for which the etiology is electrical dysfunction. ${ }^{6}$

Prior to management of monoamine RND, the amount of nutrients entering the brain is normal but it is not high enough to facilitate synthesis of monoamines at the levels needed to allow the OCT to function up to the required flow potentials encoded in the transporter. 


\section{Materials and methods}

The primary forces responsible for establishing monoamine levels throughout the body are synthesis, metabolism, and transport. Transport dominates with its control and regulation over synthesis and metabolism. ${ }^{1,8}$ The first step in the RND management protocol is simultaneous administration of serotonin and dopamine amino acid precursors in dosing values great enough to place the monoamine system into the competitive inhibition state. Then, 1 week later, a urine sample is obtained, monoamine assays are performed, and MTO interpretation is done. This enables a proper decision on the modification of amino acid dosing values in order to achieve both the serotonin and dopamine in the optimal phase 3 ranges. ${ }^{3,4,6,12}$

If the MTO-guided amino acid dosing value changes do not yield the desired results in 1 week, another specimen is obtained and submitted for additional MTO-guided dosing change recommendations. The optimal phase 3 ranges of serotonin and dopamine are achieved with the benefit of MTO. This is a complex task because, in the competitive inhibition state, changing one amino acid precursor changes all components of the equation shown in Figure 2.,4,6,12

Two or more urinary serotonin and dopamine assays, performed on different days while taking different amino acid dosing values, are required for absolute MTO verification of phases and dosing recommendations. The patient must be taking monoamine precursors in significantly varied doses for five or more days continuously to allow for equilibration of the system to the dosing change. The results of these serial assays are then compared to determine the change in urinary serotonin and dopamine levels in response to the change in amino acid precursor dosing values. , $^{3,4,6,12}$

At the initial visit it is recommended that the following adult amino acid dosing values be initiated: L-cysteine $4500 \mathrm{mg}$, L-tyrosine $3000 \mathrm{mg}$, vitamin C $1000 \mathrm{mg}$, L-lysine $500 \mathrm{mg}$, 5-HTP $300 \mathrm{mg}$, calcium citrate $220 \mathrm{mg}$, vitamin B6 $75 \mathrm{mg}$, folate $400 \mu \mathrm{g}$, and selenium $400 \mu \mathrm{g}$. The pediatric dosing values $(<17$ years) are half the adult dosing values. A full discussion of the scientific basis for each of these amino acid and cofactor nutrients is covered in previous writings by the authors. A brief overview is as follows:

L-tyrosine and 5-HTP are dopamine and serotonin precursors, respectively. Vitamin C, vitamin B6, and calcium citrate are cofactors required in the synthesis of serotonin and/or dopamine. Folate is required for optimal synthesis of sulfur amino acids. Selenium is given in response to the ability of cysteine to concentrate methylmercury in the central nervous system. L-lysine prevents loose hair follicles in a bariatric medical practice. L-cysteine is administered to compensate for L-tyrosine-induced depletion of sulfur amino acids. ${ }^{3,4,6,12}$

The literature verifies that baseline monoamine testing in the endogenous state, prior to starting monoamine amino acid precursors of serotonin and dopamine, is of no value due to lack of reproducibility when monoamine testing is performed on multiple days from the same subject. Therefore, baseline testing has no place in monoamine-related RND management. ${ }^{3,4,6,12}$

In the competitive inhibition state, laboratory testing has reproducibility on successive test dates. MTO can assist in selecting the appropriate dose of the respective amino acid precursors to achieve the required transporter flow of monoamines and amino acids for optimal RND management. ${ }^{3,4,6,12}$

\section{Three-phase transporter response}

When postsynaptic damage compromises electrical flow at that postsynaptic location, the OCT is encoded with optimal monoamine transporter configuration and flow rates to compensate for the damage. When the monoamine flows are optimized immediately above the phase 2 /phase 3 inflection point, as discussed in this section, there is optimal restoration of postsynaptic electrical flow. However, when a significant RND exists, encoded OCT needs cannot be met by dietary intake alone, and this is the basis of the RND. ${ }^{1-13}$

In the competitive inhibition state, three phases of OCT subtype 2 (OCT2) transporter response are observed. The status of monoamines in the endogenous state may be referred to as phase 0 . In phase 0 , the serotonin or dopamine entrance gates are at maximum closure, although still partially open, and the concentrations of monoamine presenting at the transporter are too low for the entrance gate to impact access of the monoamine to the transporter. In phase 0 , the monoamines simply access the OCT2 without restriction. Since urinary monoamine levels are a measurement of monoamines not transported by the OCT2, urinary monoamine levels are random in phase 0 , not affected by the entrance gate or transporter. $^{1-13}$

Proper use of MTO deciphers the optimal flow of serotonin and dopamine as established by amino acid precursor administration which is encoded in OCT2 by the damaged system. Proper implementation of MTO revolves around identification of the phase 1 , phase 2 , and phase 3 of both urinary serotonin and dopamine responses during administration of varied amino acid precursor dosing values (the competitive inhibition state). While an experienced interpreter may often be able to determine the serotonin and dopamine 
phases with one test, a high degree of certainty exists only when two urinary monoamine assays are compared while taking varied amino acid dosing values. Referring to Figure 6 , in phase 2 , the urinary serotonin and dopamine levels are low (serotonin $<80 \mu \mathrm{g}$ and dopamine $<475 \mu \mathrm{g}$ of monoamine per $g$ of creatinine). In phase 1 , there is an inverse relationship between amino acid dosing and urinary monoamine levels. In phase 3 , there is a direct correlation between amino acid dosing values and urinary monoamine levels on assay. The amino acid dosing values where the phase inflection points occur is highly variable and unique to each individual. ${ }^{1-13}$

Assayed urinary serotonin and dopamine values are reported in $\mu \mathrm{g}$ of monoamine per $\mathrm{g}$ of creatinine in order to compensate for fluctuations in urinary specific gravity. The phase 3 optimal range for urinary serotonin is defined as $80-240 \mu \mathrm{g}$ of serotonin per $\mathrm{g}$ of creatinine. The phase 3 optimal range for urinary dopamine is defined as 475-1100 $\mu \mathrm{g}$ of dopamine per $\mathrm{g}$ of creatinine. ${ }^{1-13}$ Urine samples are usually collected 6 hours prior to bedtime, with 4 pm being the most frequent collection time point. For most patients, 6 hours before bedtime is the diurnal low point of the day. ${ }^{1-13}$

\section{Organic cation transporters}

The authors have published numerous peer-reviewed articles on the topic of in situ MTO. ${ }^{1-13}$ These publications outlined the novel first and only in situ methodology for OCT functional status determination of encoded transporter optimization in humans. This paper establishes the novel RND etiology and traits associated with chronic monoamine-associated diseases and regulatory dysfunctions, ie, postsynaptic damage-induced electrical compromise and the resultant relative RND.

The monoamines and their amino acid precursors are moved across cell walls by complex molecules known as transporters. Depending on their orientation with the cell

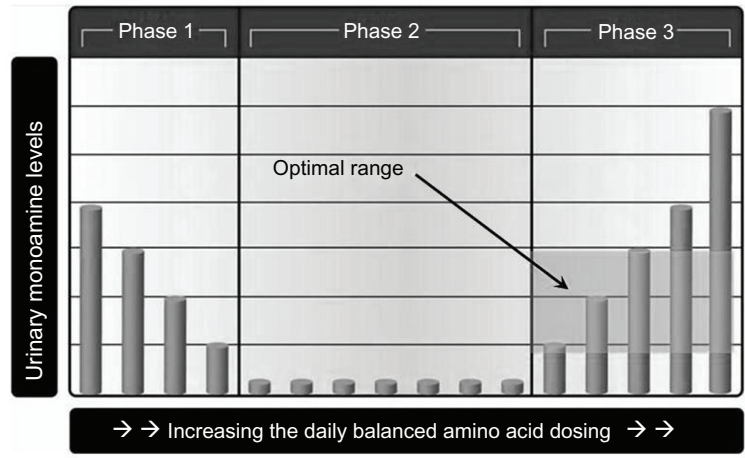

Figure 6 The core part of monoamine transporter optimization, ie, the three phases of transporter response to varied amino acid precursor dosing values. wall, transporters may move these substances in or out of the cells. ${ }^{1}$

The three primary actions that determine monoamine neurotransmitter levels everywhere in the body are synthesis, metabolism, and transport. Transporters dominate and regulate synthesis and metabolism. Synthesis is dependent on transport of amino acids into the cells. Metabolism depends on transporters to move neurotransmitters into the environment where enzymes break them down. Ultimately, intercellular and extracellular (including synaptic) monoamine and amino acid precursor levels are functions of and dependent on transporters. ${ }^{1-8,11-13}$

The following key points establish synaptic monoamine neurotransmitter levels. Monoamine neurotransmitters are stored in storage vesicles found in the presynaptic neuron. When an electrical pulse travels down the presynaptic neuron, it causes the vesicles to fuse to the presynaptic neuron cell wall, at which point neurotransmitters are excreted into the synapse. This is not the controlling event that regulates synaptic neurotransmitter levels. The synaptic monoamine levels are a function of simultaneous interaction of two transporter types. High affinity transporters are found on all neurons where monoamines are synthesized. The OCT2 regulates synaptic neurotransmitter levels by transporting neurotransmitters that escape high affinity transport. It is the OCT2 that essentially fine tunes the intercellular and extracellular monoamine levels of the brain and kidneys. OCT2 are also located on the cell membrane of the presynaptic neuron. The OCT2 perform the reuptake function, whereby the neurotransmitters are returned back into the presynaptic neurons where they are stored in the vesicles, waiting to be released anew on impulse into the synapse. ${ }^{15}$

For many years, laboratories have attempted to decode results found when neurotransmitters are assayed. The primary approach has been to determine simply whether levels were high or low. This did not work because it did not take into account the effects of transporters. This high/low approach to assay interpretation, as a guide to amino acid dosing values, was no more effective than simply giving amino acid precursors randomly. ${ }^{5,7,11}$

There are three specific items ${ }^{1-13}$ that allow for the validity of MTO:

- The various subtypes of transporters are "identical and homologous" throughout the body.

- OCT encoding occurs in an identical and homologous manner that facilitates raising levels of monoamines to establish levels high enough to relieve symptoms.

- Most importantly, OCT2 are found in only a few places in the body, mainly the kidneys and synapses of 
the brain; they are encoded identically and enable MTO determination to be an effective tool for establishing the optimal levels of amino acid precursor administration.

Based on in situ OCT observations, when the patient is suffering from chronic monoamine neurotransmitter-related disease, MTO is the only method available that allows for establishment of balanced serotonin and dopamine neurotransmitter levels needed to compensate optimally for the defective electrical flow in the brain and to relieve the RND induced by postsynaptic damage. ${ }^{1-13}$

Most patients, by history, are simultaneously suffering from three or more monoamine deficiency diseases. ${ }^{9}$ This etiology is consistent with multifocal RND. The entire clinical picture presents as multiple monoamine-related disease, but needs to be managed as a single problem with one etiology, ie, a monoamine RND relating to suboptimal function of OCT2.,

The MTO defines:

- The phase of serotonin and dopamine in OCT transport in the competitive inhibition state;

- the status of the serotonin and dopamine OCT entrance gates;

- the status of serotonin and dopamine OCT lumen saturation; and

- the OCT balance status between the monoamines and their amino acid precursors. ${ }^{1-13}$

All four of these functions are critical to determining the following in the competitive inhibition state:

- Optimal dosing values of serotonin and dopamine amino acid precursors.

- Facilitation of optimal transport of serotonin and catecholamines.

\section{Results}

The results shown in the following tables are from urinary monoamine assays which demonstrate the extreme individual variability of serotonin and dopamine precursor needs in monoamine-related RND management under the guidance of MTO in order for both serotonin and dopamine to achieve the phase 3 optimal ranges.

All three subjects in Tables 1-3 were suffering from depression with no other monoamine-related RND states present. In all three cases, when both serotonin and dopamine were established in the phase 3 optimal ranges, relief of depression symptoms was obtained. All three patients noted no relief of symptoms until both the serotonin and dopamine were established in these ranges.

The US Department of Agriculture recommended daily allowances are intended for a normal population to meet minimal daily nutrient needs, ie, to prevent absolute nutritional deficiencies. Proper management of RND is intended to be under the care of a physician because achieving a balance of neurotransmitters may require administration of nutrients in dosing values which are well above the US Department of Agriculture recommended daily allowances. ${ }^{16}$

Serotonin and dopamine amino acid dosing required to meet encoded optimal monoamine transporter flow varies greatly over large dose ranges. There is no relationship, from patient to patient, between the ultimate dosing values of serotonin and dopamine precursors required to establish serotonin and dopamine concentrations at the optimal flow encoded in the OCT.

The following ranges were extracted from a database containing over 2.4 million patient-days of amino acid management experience where monoamine-related RND were optimized with MTO. The effective therapeutic range was defined as the amino acid dosing values, within two standard deviations of the mean, that were associated with serotonin or dopamine in the phase 3 optimal range. Excluded from the data were patients suffering severe postsynaptic dopamine injury, such as Parkinson's disease and restless leg syndrome.

- The daily effective therapeutic range of 5-HTP as evidenced by a phase 3 serotonin in the 80 to $240 \mu \mathrm{g} / \mathrm{g}$ creatinine range was found to be $>0 \mathrm{mg}$ to $2400 \mathrm{mg}$.

- The daily L-dopa effective therapeutic range as evidenced by phase 3 dopamine in the 475 to $1100 \mu \mathrm{g} / \mathrm{g}$ creatinine range was found to be $>0 \mathrm{mg}$ to $2100 \mathrm{mg}$.

- The daily effective therapeutic range of L-tyrosine as evidenced by dopamine response to L-dopa administration was found to be $>0 \mathrm{mg}$ to $14,000 \mathrm{mg}$.

The dosing values of 5-HTP, L-dopa, and L-tyrosine are independent of each other. Some variability in the high-end range values may occur when individual RND-associated disease states are examined versus this entire group of diseases. Using L-tyrosine as an example, dosing values of $14,000 \mathrm{mg}$ per day were unknown in the literature prior to this research. However, when amino acid dosing values are established with MTO, these seemingly high doses are uniformly well tolerated by patients, because electrical flow and the system revert back to normal.

\section{Discussion}

The OCT2 of the brain fine tunes monoamine neurotransmitter levels. The OCT2 of the brain and kidneys are identical and homologous and share certain specific traits, including being genetically identical with regard to DNA sequencing. ${ }^{16}$ In order to understand the significance of the amino acid 
Table I Patient with depression suffering from postsynaptic serotonin neuronal damage, as evidenced by the level of 5-HTP required to control the RND

Urinary serotonin and dopamine reported in $\mu \mathrm{g}$ monoamine per $\mathrm{g}$ of creatinine

Amino acids ( $\mu$ g/day)

\begin{tabular}{llllllll}
\hline Date & Serotonin & Serotonin phase & Dopamine & Dopamine phase & 5-HTP & L-dopa & L-tyrosine \\
\hline$I I / I / 20 I I$ & 873 & 1 & 536 & 3 & 300 & 240 & 3000 \\
$I / \mid 8 / 20 I I$ & 27 & 2 & 986 & 3 & 600 & 240 & 4000 \\
$\mid 2 / 4 / 20 I I$ & 187 & 3 & $49 I$ & 3 & 900 & 120 & 5000 \\
\hline
\end{tabular}

Abbreviations: 5-HTP, 5-hydroxytryptophan; L-dopa, L-3,4-dihydroxyphenylalanine; RND, relative nutritional deficiency.

dosing value variables found in Tables $1-3$, it is necessary to review OCT2 transporter physiology.

Serotonin and dopamine transport across the basolateral membrane of the proximal convoluted renal tubule cells is identical to the mechanism of action in the brain. A high affinity transporter is involved and the OCT2 transports and fine tunes monoamine neurotransmitter levels not transported by the high affinity transporters. ${ }^{17}$ The urinary assays of Tables $1-3$ represent monoamines that are newly synthesized in the proximal convoluted renal tubule cells and are not transported across the basolateral membrane by the high affinity OCT 2 system. These newly synthesized monoamines, not transported by the basolateral transporter system, are transported via the OCTN2 through the apical membrane, finally ending up in the urine. ${ }^{1,8}$

The high affinity OCT2 system in the brain primarily functions as the monoamine reuptake transporters located on the presynaptic neurons. Synaptic monoamine levels represent monoamines that have not been transported into the presynaptic neurons. This is a functional transporter characteristic of the brain and is identical and homologous to OCT2 function of the proximal renal tubule cells. The synaptic monoamine levels are analogous to the monoamine concentrations found in the urine. Monoamine reuptake inhibitor drugs interact with OCT2 transporters. ${ }^{1-13}$

When postsynaptic damage associated with compromise in the flow of electricity occurs leading to development of disease symptoms:

- An increase in synaptic monoamine levels compensates by facilitating the increased flow of electricity.
- OCT2 transporters are encoded to establish monoamine concentrations required to compensate for the problem. This is evidenced by the monoamine/amino acid dosing value variability in correlation with the clinical response of symptom resolution.

- Serotonin and dopamine amino acid precursor administration results in synaptic and urinary monoamine levels following the three phase response. ${ }^{1-13}$

As noted in Figure 6, the optimal amino acid dosing values as identified by MTO places the serotonin and dopamine in phase 3 just above the phase 2/phase 3 inflection point. In the optimal phase 3 dosing range, the OCT2 entrance gates are fully open, and the flow through the transporter has become saturated with serotonin and dopamine. This occurs at the phase 2 /phase 3 inflection point as the total amount of serotonin and dopamine presenting at the transporter entrance increases. In the competitive inhibition state, the concentration of serotonin and dopamine reported on assay is not as important as achieving proper balance of the monoamines in the optimal phase 3 ranges as defined by MTO. ${ }^{1-13}$

For example, a serotonin concentration of $230 \mu \mathrm{g} / \mathrm{g}$ creatinine may appear to be in the optimal range if only concentration values are considered. However, when this laboratory value is found to be in phase 1 , a completely different physiological state emerges, ie, one of suboptimal synaptic function and restricted monoamine access to the transporter because the system gives priority to elevating synaptic monoamine levels at the expense of optimizing monoamines stored in the presynaptic vesicles. ${ }^{1-13}$

Table 2 Patient with depression suffering from postsynaptic catecholamine neuronal damage as evidenced by the level of L-dopa required to control the RND

\begin{tabular}{|c|c|c|c|c|c|c|c|}
\hline \multicolumn{8}{|c|}{ Urinary serotonin and dopamine reported in $\mu \mathrm{g}$ monoamine per $\mathrm{g}$ of creatinine } \\
\hline \multicolumn{8}{|c|}{ Amino acids ( $\mu$ g/day) } \\
\hline Date & Serotonin & Serotonin phase & Dopamine & Dopamine phase & 5-HTP & L-dopa & L-tyrosine \\
\hline $10 / 4 / 2011$ & 3392 & 3 & 554 & I & 300 & 240 & 3000 \\
\hline $10 / 22 / 2011$ & 2343 & 3 & 283 & 2 & 150 & 480 & 1500 \\
\hline||$/ 6 / 20||$ & 216 & 3 & 694 & 3 & 37.5 & 720 & 375 \\
\hline
\end{tabular}

Abbreviations: 5-HTP, 5-hydroxytryptophan; L-dopa, L-3,4-dihydroxyphenylalanine; RND, relative nutritional deficiency. 
Table 3 Patient with depression suffering from postsynaptic serotonin and catecholamine neuronal damage as evidenced by the levels of 5-HTP and L-dopa required to control the RND

\begin{tabular}{|c|c|c|c|c|c|c|c|}
\hline \multicolumn{8}{|c|}{ Urinary serotonin and dopamine reported in $\mu \mathrm{g}$ monoamine per $\mathrm{g}$ of creatinine } \\
\hline \multicolumn{8}{|c|}{ Amino acids ( $\mu$ g/day) } \\
\hline Date & Serotonin & Serotonin phase & Dopamine & Dopamine phase & 5-HTP & L-dopa & L-tyrosine \\
\hline $8 / 4 / 2011$ & 1496 & I & 362 & 2 & 300 & 240 & 3000 \\
\hline $8 / 22 / 2011$ & 1288 & I & 178 & 2 & 600 & 360 & 4000 \\
\hline $9 / 6 / 2011$ & 1213 & I & 86 & 2 & 900 & 480 & 5000 \\
\hline $10 / 4 / 2011$ & 761 & I & 152 & 2 & 1200 & 720 & 6000 \\
\hline $10 / 22 / 2011$ & 364 & I & 187 & 2 & 1500 & 960 & 7000 \\
\hline $11 / 6 / 201 \mid$ & 168 & I & 248 & 2 & 1800 & 1200 & 8000 \\
\hline $\mid \mathrm{I} / 23 / 201 \mathrm{I}$ & 64 & 2 & 417 & 2 & 2100 & 1440 & 9000 \\
\hline$|2 / 9 / 20| \mid$ & $16 \mid$ & 3 & 513 & 3 & 2400 & 1440 & 10,000 \\
\hline
\end{tabular}

Abbreviations: 5-HTP, 5-hydroxytryptophan; L-dopa, L-3,4-dihydroxyphenylalanine; RND, relative nutritional deficiency.

Synaptic monoamine levels and presynaptic vesicle monoamine levels are a function of OCT2 functional status. When compromise in electrical flow is present, the OCT2 is encoded with the monoamine transporter flow characteristics required for optimal flow through the presynaptic, synaptic, and postsynaptic systems. This novel transporter encoding variability is vigorously displayed in Tables 1-3, where MTO defines the required optimal serotonin and dopamine amino acid precursors..$^{1-13}$

A hypothesis of this research states that, in the endogenous state, when postsynaptic neuron damage occurs, a point is reached where the transporters are unable to alter the flow of available monoamines sufficiently to keep the electrical flow at a level great enough for the system to function normally and the patient to be symptom-free. When the total monoamine concentration in the system is normal but too low for the transporters to optimize synaptic levels, it is the result of an RND of serotonin and/or dopamine; this requires resolution with a nutrient-based amino acid precursors approach any time low or inadequate levels of monoamine neurotransmitters exist. ${ }^{1-13}$

One of the foundations for monoamine-associated RND and the ability to compensate for this problem is illustrated in Tables 1-3. The body responds to postsynaptic neuron damage by encoding the OCT 2 in a unique and individualized manner that facilitates synaptic monoamine compensation. However, monoamine levels that are high enough to allow the OCT2 to compensate are not achievable on a regular diet, so the system languishes in the phase 0 state. ${ }^{1-13}$

By administration of properly balanced nutrients (amino acids), optimal synaptic monoamine levels are established and relief of symptoms and/or proper regulation of function occur. As discussed in the Results section, the amino acid dosing values required to achieve optimal OCT2 function vary greatly and are very individualized. Once the proper amino acid dosing needed to relieve symptoms is found, it becomes that patient's standard nutrient intake requirement to compensate for the RND unless further postsynaptic damage is experienced. ${ }^{1-13}$

The site of postsynaptic neuron damage in the brain dictates the nature of the RND and the monoamine-associated disease symptoms that are manifest. With Parkinson's disease, damage occurs in the dopamine neurons of the substantia nigra. Patients suffering chronic depression sustain postsynaptic damage to the regions of the brain that control affect and mood. This could be a damage-associated RND of the serotonin, dopamine, or norepinephrine postsynaptic neurons or any combination thereof (Tables $1-3){ }^{1-13}$

The amino acid dosing values found in Table 3 deserve some additional reflection. The dosing values of 5-HTP and L-tyrosine are novel, and much larger than reported in the previous literature. The dosing value of L-dopa for this nonParkinson's patient is relatively large as well. Administration of the novel amino acid dosing values needed to properly address RND which are this large, with successful resolution of symptoms, would not be possible or considered without MTO.

Side effects and adverse reactions due to imbalanced administration of amino acid dosing values of this magnitude without MTO guidance would prohibit dosing values such as this, effectively establishing an amino acid dosing barrier. Further, without MTO, there is no objective amino acid dosing value guidance in addressing the RND; it is a random event in an environment where individual needs vary on a large scale and the dosing needs of serotonin and dopamine precursors are independent of each other. When serotonin and dopamine levels are increased to levels required to address the RND and proper balance is achieved with MTO 
guidance, these amino acid dosing values, such as found in Table 3, are exceptionally well tolerated and generate the desired result of safely alleviating symptoms. The key is proper balance. MTO reveals that if side effects and adverse reactions occur during amino acid administration, they are not due to a specific amino acid; rather, imbalance between the serotonin and dopamine systems is the cause. The lack of unmanageable side effects, such as those observed when only L-dopa is administered for management of Parkinson's disease, is attributable to the balanced administration of the precursors which restore neuronal electrical flow and system function to normal. ${ }^{1-13}$

Administration of proper levels of amino acids does not make the patient high or euphoric. In response to establishing the serotonin and dopamine in the phase 3 optimal ranges, symptoms resolve and the patient simply feels normal. What matters is getting the required levels of balanced amino acids into the system to compensate for the RND associated with the electrical defect under the guidance of MTO without regard to how large the amino acid dosing value has become, as long as the need is indicated. ${ }^{1-13}$

\section{Amino acid-induced RND}

An RND of the nondominant system occurs when there is an improper balance between the serotonin and dopamine amino acid precursors. The three primary forces that regulate concentrations of centrally acting monoamines throughout the body are synthesis, metabolism, and transport. The serotonin and catecholamine systems are so heavily intertwined in the competitive inhibition state that they need to be managed as one system under MTO guidance to achieve optimal results. Changes to one component of either system will affect all components of both systems in a predictable manner. ${ }^{8}$

Giving only 5-HTP or only L-dopa or improperly balanced serotonin and dopamine amino acid precursors (Figure 2) will, over time, create many problems which result in needless patient suffering from suboptimal monoamine levels, increased side effects, and false expectations during medical care. ${ }^{8}$

Unbalanced administration of serotonin and dopamine amino acid precursors causes:

- One system to dominate over the other system in synthesis, transport, and metabolism (see Figures 3-5) leading to depletion of the nondominant system. ${ }^{8}$

- Increased incidence of side effects due to administration of improperly balanced amino acids. ${ }^{8}$
- The inability to achieve the amino acid dosing values needed to optimize MTO fully, which prevents both optimal management of the RND and restoration of proper postsynaptic neuron flow. ${ }^{8}$

\section{latrogenic or drug-induced RND}

Depletion of monoamine neurotransmitters is known in the literature to be associated with administration of reuptake inhibitors. Reuptake inhibitors are not just prescription drugs used for treatment of depression and attention-deficit disorder, but are also available as street drugs, such as amphetamines, "Ecstasy," and methamphetamine. Reuptake inhibitors deplete monoamines via their mechanism of action, which induces an RND. All amphetamines also have serious neurotoxic potential and are fully capable of inducing a neurotoxin-associated RND, with postsynaptic neuron damage in addition to the reuptake inhibitor-driven RND. Selective serotonin reuptake inhibitors are also known to decrease serotonin synthesis, leading to a drug-induced RND. The nonspecific reuptake inhibitor amitriptyline (a tricyclic antidepressant) is known to deplete norepinephrine, leading to a drug-induced RND. ${ }^{13}$

A series of illustrations (Figures 7-9) have been posted on The National Institute on Drug Abuse's website. These figures show how reuptake inhibitors deplete monoamine neurotransmitters leading to the induction of an RND. $^{13}$

Drugs that work with neurotransmitters do not function properly if there are not enough synaptic neurotransmitters available. The end stage of reuptake inhibitor-induced

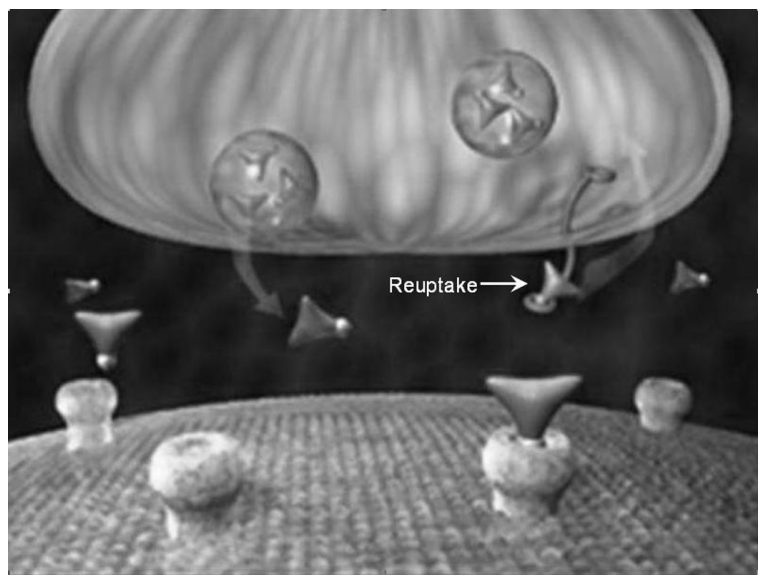

Figure 7 Prior to reuptake inhibitor treatment, inadequate levels of neurotransmitters in the synapse cause a disease-associated relative nutritional deficiency leading to compromised electrical flow through the postsynaptic neurons resulting in suboptimal regulation of function and/or development of symptoms. 


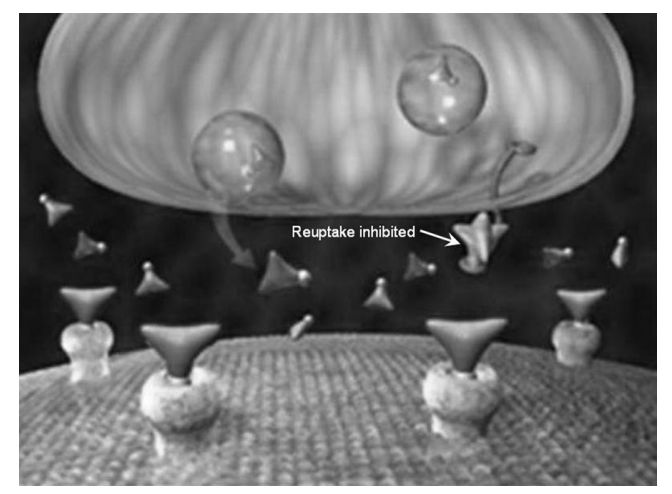

Figure 8 Administration of reuptake inhibitors blocks monoamine transport back into the presynaptic neurons. This leads to a net redistribution of neurotransmitter molecules from the presynaptic neuron to the synapse. The increased synaptic level of monoamines increases post-synaptic flow of electricity leading to restoration of adequate regulation of function and/or relief of symptoms.

RND occurs when there is severe depletion of the neurotransmitters:

- Drug stops working.

- Discontinuation syndrome is so strong that the patient cannot discontinue the drug even though there is no perceived benefit.

- Suicidal ideation develops.

When this happens, administration of properly balanced serotonin and dopamine amino acid precursors will correct the RND, restore the effects of the drug, and restore the normal functioning of the system. ${ }^{13}$

\section{Disease-induced RND}

Inadequate flow of postsynaptic electricity is associated with virtually all chronic monoamine-related diseases.

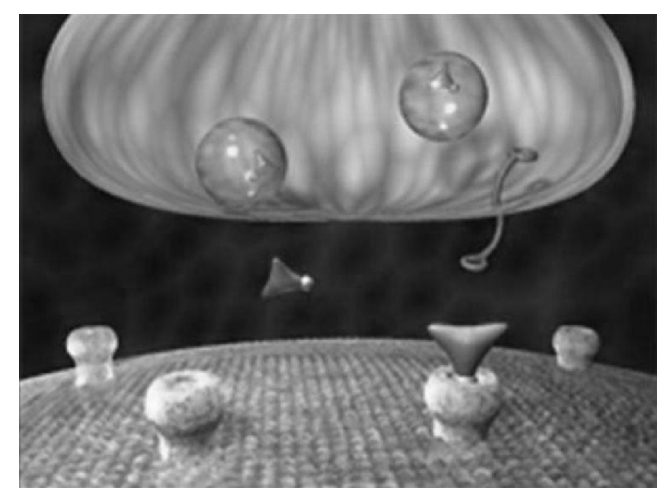

Figure 9 The drug-induced relative nutritional deficiency. When the monoamines are in the vesicles of the presynaptic neuron, they are not exposed to the enzymes that catalyze metabolism (monoamine oxidase and catechol-O-methyltransferase). They are safe from metabolism. When they are relocated outside the vesicles of the presynaptic neuron, they are exposed to these enzymes at a greater frequency. Reuptake inhibitors create a mass migration of monoamines causing increased metabolic enzyme activity and metabolism of monoamines. This leads to the druginduced relative nutritional deficiency if significant amounts of balanced serotonin and dopamine precursors are not coadministered with the reuptake inhibitor.
In all cases where synaptic monoamine levels are normal but not adequate such as states where low or inadequate levels of monoamine neurotransmitters occur, there is a monoamineassociated RND. Even with the use of reuptake inhibitor drugs, proper management of these problems involves addressing the RND by administering the monoamines and their amino acid precursors. Optimization can only be achieved with MTO.

The ability of MTO to address monoamine-related RND is so definitive that proper implementation leads, with absolute certainty, to determining whether monoamine neuronal electrical dysfunction is a component of the disease picture. The examples below illustrate how proper application of monoamine transport optimization can lead to recognition and resolution of the RND and also allow for observation of other problems not clearly anticipated as disease etiologies.

\section{Major affective disorder}

Chronic major affective disorder (depression) has an RND present which leads to monoamine levels in the central nervous system being too low to achieve optimal postsynaptic flow of electricity. Properly balanced amino acid precursors are necessary; dietary nutrient intake alone is not sufficient to establish high enough monoamine levels to optimize transporter-dependent synaptic monoamines. ${ }^{9}, 12$

Contrary to the popular assertion that 5-HTP is indicated for depression, MTO reveals that use of only 5-HTP for depression is contraindicated. Many patients with depression respond only to drugs with dopamine and/or norepinephrine reuptake inhibition properties. Administration of only 5-HTP leads to an amino acid-induced RND of the catecholamines which leads to exacerbation of depression, especially in patients whose depression is dominated by catecholamine dysfunction. Use of only 5-HTP depletes catecholamines. When catecholamine depletion is great enough, any clinical benefits initially observed with the administration of 5-HTP will be no longer present. ${ }^{1-13}$

Reuptake inhibitors have only marginal effectiveness in addressing the symptoms associated with depression and no ability to address the etiology of the RND. In doubleblind studies of major affective disorder, only $7 \%-13 \%$ of patients achieve symptom relief greater than placebo. Drug administration reveals subgroups of patients suffering from major affective disorder who achieve greater efficacy with a serotonin, dopamine, or norepinephrine reuptake inhibitor or combination. The area of the brain that controls affect involves interactions of all three of these monoamines. The mechanism and site of action in the affected area of the 
brain will dictate which of these monoamines are primarily involved. While recognizing that any of several monoamines may be involved while displaying identical symptomatology, the exact determination of which ones are primarily involved is not required. The MTO approach simultaneously optimizes levels of all three of these monoamines in transport, based on interpretation of information encoded in the transporters. ${ }^{9,12,13}$

Standard management for many patients with depression includes prescribing reuptake inhibitor antidepressants. If proper levels of nutrients are not administered concomitantly with the drug, monoamine neurotransmitter depletion may and often does occur, leading to a drug-induced RND., ${ }^{9}$

Two primary types of depression are recognized here, ie, major affective disorder and bipolar disorder cycling on the depressive pole (bipolar depression). As was previously noted in the literature, when OCT serotonin and dopamine levels were established with MTO in the optimal phase 3 ranges, all subjects whose depression did not resolve were suffering from bipolar depression. ${ }^{12}$

A review of the clinical history prior to initiation of management revealed that these patients had no response to bipolar medications in the past and had no response when amino acids were optimized. These patients had all been treated with a mood-stabilizing drug without success. This is an RND that requires both serotonin and dopamine to be placed in the phase 3 optimal ranges before the effects of mood-stabilizing drugs are observed. When the amino acid dosing values required for MTO were achieved and a mood-stabilizing drug (lithium $300 \mathrm{mg}$ twice a day or valproic acid $250 \mathrm{mg}$ two or three times a day) was added, $>98 \%$ of cases experienced resolution of depressive bipolar symptoms in 1-3 days. These bipolar depressive patients were suffering from damage at a central nervous system site distinctly different from that of major affective disorder. Bipolar patients require addition of a mood-stabilizing drug that had previously yielded no benefit but became effective once the RND was properly addressed with the aid of MTO. ${ }^{12}$

\section{Parkinson's disease}

Standard medical management of Parkinson's disease uses L-dopa and carbidopa. This approach literally turns into a case study of how many iatrogenic side effects and adverse reactions can be amassed during amino acid mismanagement of patients. Under this approach, traditionally there is a total disregard for the interactions of L-dopa and the peripheral monoamine status induced by carbidopa (see Figure 2). ${ }^{6}$
L-dopa is recognized as the most effective management option for Parkinson's disease, but is generally not used firstline due to the exceptionally large amount of iatrogenically induced significant side effects and problems that evolve over time. Previous literature published by the authors asserts that virtually all of the problems associated with administration of L-dopa and/or carbidopa are caused by iatrogenic mismanagement of the large number of RND associated with the disease, L-dopa, and/or carbidopa. These RND involve all three major classes of RND, ie, disease-associated, amino acid-induced, and drug-induced. ${ }^{6}$

The Parkinson's disease-associated RND is characterized by damage to the postsynaptic dopamine neurons of the substantia nigra. The extremely high synaptic dopamine levels required to restore normal flow of electricity cannot be established by dietary intake alone. ${ }^{6}$

Parkinson's disease-associated RND management may require L-dopa dosing values up to 200 times greater than the needs of other monoamine disease processes, as high as 25,000 mg per day. MTO reveals that the OCT2 are encoded to elevate synaptic dopamine vigorously, to the point that serotonin is excluded from the transporter leading to development of a Parkinson's disease-associated serotonin RND. Other Parkinson's disease RND include norepinephrine and epinephrine which are dependent on dopamine levels for synthesis and are inadequate when the disease is present. ${ }^{6}$

The three primary monoamine RND associated with Parkinson's disease are shown in Figure 10. The only practical way to increase the depleted levels of monoamines and amino acids noted in Figure 10 is by administration of amino acid precursors guided by MTO. ${ }^{6}$

Administration of L-dopa is also known to induce RND associated with L-tyrosine, sulfur amino acids, L-tryptophan,

\begin{tabular}{lccc} 
& $\begin{array}{c}\text { Status in } \\
\text { Parkinson's } \\
\text { disease }\end{array}$ & $\begin{array}{c}\text { Status with } \\
\text { L-dopa Rx }\end{array}$ & $\begin{array}{c}\text { Status with } \\
\text { Carbidopa Rx }\end{array}$ \\
\hline Serotonin (Central) & Depleted & Further depleted & \\
\hline Dopamine (Central) & Depleted & & \\
\hline Norepinephrine (Central) & Depleted & & \\
\hline Epinephrine (Central) & Depleted & & Further depleted \\
\hline Serotonin (Peripheral) & Depleted & Further depleted & Further depleted \\
\hline Dopamine (Peripheral) & Depleted & & Further depleted \\
\hline Norepinephrine (Peripheral) & Depleted & & Further depleted \\
\hline Epinephrine (Peripheral) & Depleted & & \\
\hline L-tyrosine & & Depleted & \\
\hline Tyrosine Hydroxylase & Depleted & & \\
\hline L-tryptophan & & Depleted & \\
\hline 5-Hydroxytryptophan & & Depleted & \\
\hline Sulfur amino acids & & Depleted & \\
\hline
\end{tabular}

Figure 10 Basis for multiple relative nutritional deficiencies associated with Parkinson's disease. 
5-HTP, and serotonin (see Figure 2). ${ }^{6}$ The following are previously published categories of L-dopa-associated problems that are now correlated with an L-dopa-associated RND. ${ }^{6}$

\section{Serotonin-related RND induced by L-dopa}

A serotonin RND is the primary reason the L-dopa quits functioning (tachyphylaxis, ie, L-dopa stops working). ${ }^{6} \mathrm{~L}$-dopa tachyphylaxis is precipitated by depletion of serotonin when dominant levels of L-dopa are administered. Administration of 5-HTP to restore the balance guided by MTO is required to manage this RND properly.

\section{RND-induced transport imbalance between serotonin and dopamine}

This RND-related problem is responsible for a number of side effects associated with the administration of L-dopa in a dominant manner, ie, nausea, vomiting, anorexia, weight loss, decreased mental acuity, depression, psychotic episodes including delusions, euphoria, pathological gambling, impulse control, confusion, dream abnormalities including nightmares, anxiety, disorientation, dementia, nervousness, insomnia, sleep disorders, hallucinations and paranoid ideation, somnolence, memory impairment, and increased libido. $^{6}$

An imbalance in the administration of serotonin and dopamine amino acid precursors is responsible for all of the above listed side effects and adverse reactions. MTO is required when serotonin precursors are started in combination with L-dopa. Several of the side effects, such as nausea, may be caused by administering the serotonin amino acid precursor at levels that are either too high or too low. Since the status of serotonin could be too high or too low, the level cannot be empirically determined and MTO is required.

\section{L-tyrosine RND}

L-tyrosine RND may contribute to the associated on-off effect, motor fluctuations, or dopamine fluctuations. ${ }^{6}$ MTO has identified fluctuations in dopamine transport that respond to L-tyrosine administration. The etiology of this phenomenon remains unknown.

\section{L-dopa-induced sulfur amino acid RND}

L-dopa-induced sulfur amino acid RND is associated with bradykinesia (epinephrine depletion implicated), akinesia, dystonia, chorea, extrapyramidal side effects, fatigue, abnormal involuntary movements, and depletion of glutathione, potentiating further the dopamine neuron damage done by neurotoxins. ${ }^{6}$ Patients with Parkinson's disease as a group have significantly depleted sulfur amino acid levels, leading to an associated RND which is exacerbated by administration of L-dopa. Neurotoxins are the leading etiology of postsynaptic dopamine damage in Parkinson's disease. Glutathione is the body's most powerful toxin-neutralizing agent and is synthesized from sulfur amino acids. When a sulfur amino acid RND occurs, it may accelerate the progression of Parkinson's disease due to increased susceptibility to further neurotoxic insult.

\section{Carbidopa-induced peripheral serotonin and catecholamine RND}

Carbidopa-induced peripheral serotonin and catecholamine depletion cause RND that are associated with numerous side effects and adverse reactions, ie, dyskinesia, glossitis, leg pain, ataxia, falling, gait abnormalities, blepharospasm (which may be taken as an early sign of excess dosage), trismus, increased tremor, numbness, muscle twitching, peripheral neuropathy, myocardial infarction, flushing, oculogyric crises, diplopia, blurred vision, dilated pupils, urinary retention, urinary incontinence, dark urine, hoarseness, malaise, hot flashes, sense of stimulation, dyspepsia, constipation, palpitation, fatigue, upper respiratory infection, bruxism, hiccups, common cold, diarrhea, urinary tract infections, urinary frequency, flatulence, priapism, pharyngeal pain, abdominal pain, bizarre breathing patterns, burning sensation of tongue, back pain, shoulder pain, chest pain (noncardiac), muscle cramps, paresthesia, increased sweating, falling, syncope, orthostatic hypotension, asthenia (weakness), dysphagia, Horner's syndrome, mydriasis, dry mouth, sialorrhea, neuroleptic malignant syndrome, phlebitis, agranulocytosis, hemolytic and nonhemolytic anemia, rash, gastrointestinal bleeding, duodenal ulcer, HenochSchonlein purpura, decreased hemoglobin and hematocrit, thrombocytopenia, leukopenia, angioedema, urticaria, pruritus, alopecia, dark sweat, abnormalities in alkaline phosphatase, abnormalities in serum glutamic oxaloacetic transaminase (aspartate aminotransferase), serum glutamic pyruvic transaminase (alanine aminotransferase), abnormal Coombs' test, abnormal uric acid, hypokalemia, abnormalities in blood urea nitrogen, increased creatinine, increased serum lactate dehydrogenase, and glycosuria. ${ }^{6}$

The problem in this category is a carbidopa-induced RND of peripheral serotonin and catecholamines, and is best managed by not using carbidopa in the first place. It is not needed when MTO is properly utilized. Carbidopa was originally employed in an effort to control the nausea associated with L-dopa administration, a side effect and RND manageable by MTO. 
Carbidopa inhibits peripheral synthesis of serotonin and catecholamines by L-aromatic amino acid decarboxylase. In the process, peripheral monoamines develop an associated RND with a plethora of symptoms (see above). By far, the largest group of RND-related side effects and adverse reactions in the management of Parkinson's disease are due to carbidopa-induced RND. All of the reasons for which carbidopa is added to L-dopa can be safely and easily managed with MTO. ${ }^{6}$

\section{Attention-deficit hyperactivity disorder RND}

Double-blind, placebo-controlled studies of attention-deficit hyperactivity disorder (ADHD) have revealed drug efficacy (reuptake inhibitor and stimulant) greater than placebo in $14 \%-41 \%$ of patients studied. ${ }^{4}$

Drug treatment revolves around administration of reuptake inhibitors, such as atomoxetine (a norepinephrine reuptake inhibitor) and stimulants. The stimulants are divided into two classes, ie, amphetamine and nonamphetamine. Both classes have dopamine and norepinephrine reuptake properties, along with the potential for neurotransmitter depletion. ${ }^{4}$ ADHD patients are exposed to drug-induced RND:

- from reuptake inhibitors which deplete neurotransmitters

- from the amphetamines (neurotoxins) which cause brain damage.

All of this is avoided with the amino acid administration approach guided by MTO, because ADHD responds well to this RND. ${ }^{4}$ A previous study indicated that pediatric ADHD management with amino acid administration guided by MTO which addressed the associated monoamine RND may be more effective than methylphenidate and atomoxetine. ${ }^{4}$

\section{Crohn's disease RND}

Crohn's disease is a prototype for studying genetically associated RND. There is a known genetic defect of OCTN1 and OCTN2 transporters in the proximal and distal colon of patients suffering from Crohn's disease. As with the OCT, the OCTN is capable of transporting organic cations, including serotonin, dopamine, and their precursors. In Crohn's disease, the serotonin content of the mucosa and submucosa of the proximal and distal colon is significantly increased. The only reasonable explanation, as verified by clinical response, is that the OCTN1 and OCTN2 genetic deficits induce increased synthesis and tissue levels of serotonin. Based on MTO with Crohn's patients, it appears that a severe imbalance between high serotonin levels and RND-associated dopamine transport, synthesis, and metabolism contributes significantly to disease symptoms. The literature suggests that much of the clinical constellation found with Crohn's disease is induced by serotonin toxicity in the colon exacerbated by dopaminerelated RND that exist simultaneously. ${ }^{3}$ Control of the disease symptoms and resolution of all gut lesions has been shown to occur with proper MTO-guided balanced amino acid dosing, without the use of any drugs and in cases where conventional drugs have had no positive effect. ${ }^{3}$

\section{Other diseases}

The rest of the diseases and regulatory functions listed in Appendix A and Appendix B share the same basic approach to diagnosis, etiology, and RND management. If a monoamine-related RND is suspected where synaptic monoamine levels are not high enough to compensate for postsynaptic electrical defects, the amino acid dosing values needed to correct the problem can be identified and achieved with MTO.

\section{Conclusion}

The authors have published multiple papers relating to MTO. In the course of further research and writing efforts, it was realized that the most basic etiological factors relating to monoamine disease had not been previously discussed, ie, the presence of RND. The purpose of this paper is to clarify how common an etiology RND is and why it needs to be considered.

Neurotoxic, traumatic, biological, and genetic components that induce permanent brain damage are real. Without an objective guidance tool such as MTO, specific problems relating to the association of these RND with this damage is not properly recognized or managed with either drugs or amino acids. Most physicians do not recognize toxicity as a cause of these diseases and few understand the existence of the common RND-based etiology. The treatment of symptoms with drugs, rather than addressing and resolving underlying RND with nutrients, leads to gross failures during management, prolonged unneeded disability, exacerbation of the disease, and morbidity.

Many things are explained by becoming cognizant of the role of chronic postsynaptic damage, as associated with RND. In double-blind studies of the treatment of depression, reuptake inhibitors are only $7 \%-13 \%$ more effective than placebo. The monoamine RND model makes sense out of that information. Reuptake inhibitors are only able to increase transporter-driven synaptic monoamine levels minimally in phase 0 which, in the longer term, may lead to monoamine depletion after the response. 
The RND models discussed in this paper have demonstrated how the damage might be related to either dopamine, norepinephrine, or serotonin neurons, or a combination of these. MTO defines the proper balance of amino acids in order to establish adequate synaptic levels of monoamines to compensate for postsynaptic damage and the electrical deficit, while relieving the etiological RND. It is the goal of this writing to stimulate interest and dialog based on these novel observations. The ability to address the cause of a problem with nutrients is more desirable than only treating the symptoms with a drug.

\section{Disclosure}

The authors report no conflicts of interest in this work.

\section{References}

1. Hinz M, Stein A, Uncini T. Discrediting the monoamine hypothesis. Int J Gen Med. 2012;5:135-142.

2. Hinz M, Stein A, Uncini T. The dual-gate lumen model of renal monoamine transport. Neuropsychiatr Dis Treat. 2010;6:387-392.

3. Hinz M, Stein A, Uncini T. Amino acid-responsive Crohn's disease: a case study. Clin Exp Gastroenterol. 2010;3:171-177.

4. Hinz M, Stein A, Uncini T. Treatment of attention deficit hyperactivity disorder with monoamine amino acid precursors and organic cation transporter assay interpretation Neuropsychiatr Dis Treat. 2011;7:31-38.

5. Hinz M, Stein A, Uncini T. Urinary neurotransmitter testing: considerations of spot baseline norepinephrine and epinephrine. Open Access Journal of Urology. 2011;3:19-24.

6. Hinz M, Stein A, Uncini T. Amino acid management of Parkinson's disease: A case study. Int J Gen Med. 2011;4:1-10.
7. Hinz M, Stein A, Uncini T. Validity of urinary monoamine assay sales under the "spot baseline urinary neurotransmitter testing marketing model". Int J Nephrol Renovasc Dis. 2011;4:101-113.

8. Hinz M, Stein A, Uncini T. APRESS: apical regulatory super system, serotonin, and dopamine interaction. Neuropsychiatr Dis Treat. 2011; $7: 1-7$.

9. Hinz M. Depression. In: Kohlstadt I, editor. Food and Nutrients in Disease Management. Boca Raton, FL: CRC Press; 2009.

10. Trachte G, Uncini T, Hinz M. Both stimulatory and inhibitory effects of dietary 5-hydroxytryptophan and tyrosine are found on urinary excretion of serotonin and dopamine in a large human population. Neuropsychiatr Dis Treat. 2009;5:227-235.

11. Hinz M, Stein A, Trachte G, Uncini T. Neurotransmitter testing of the urine: a comprehensive analysis. Open Access Journal of Urology. 2010;2:177-183.

12. Hinz M, Stein A, Uncini T. A pilot study differentiating recurrent major depression from bipolar disorder cycling on the depressive pole. Neuropsychiatr Dis Treat. 2010;6:741-747.

13. Hinz M, Stein A, Uncini T. Monoamine depletion by reuptake inhibitors. Drug Healthc Patient Saf. 2011;3:69-77.

14. CMTA Charcot-Marie-Tooth Association [homepage on the Internet]. Glenolden, PA: Charcot-Marie-Tooth Association; 2006-2011. Available from: http://www.cmtausa.org/index.php?option=com_con tent\&view=article\&id=68\&Itemid=42. Accessed February 12, 2012.

15. Andreas B, Ulrich K, Dagar M, et al. Human neurons express the polyspecific cation transporter hOCT2, which translocates monoamine neurotransmitters, amantadine, and memantine. Mol Pharmacol. 1998;54:342-352.

16. Food and Nutrition Information Center [homepage on the Internet]. USDA National Agricultural Library; updated 2012. Available from: http://fnic.nal.usda.gov/nal_display/index.php?info_center=4\&tax_ level=1\&tax_subject $=620$. Accessed February 12, 2012.

17. Wing-Kee L, Markus R, Bayram E, et. al. Organic cation transporters OCT1, 2, and 3 mediate high-affinity transport of the mutagenic vital dye ethidium in the kidney proximal tubule. Am J Physiol Renal Physiol. 2009;296:F1504-F1513. 


\section{Appendix A}

Partial listing of central nervous system monoamine dysfunction-related diseases

Parkinson's disease

Obesity

Bulimia

Anorexia

Depression

Anxiety

Panic attacks

Migraine headaches

Tension headaches

Premenstrual syndrome

Menopausal symptoms

Obsessive compulsive disorder

Obsessionality

Insomnia

Impulsivity

Aggression

Inappropriate aggression

Inappropriate anger

Psychotic illness

Fibromyalgia

Chronic fatigue syndrome

Adrenal fatigue/burnout

Hyperactivity

Attention-deficit hyperactivity disorder

Hormone dysfunction

Adrenal dysfunction

Dementia

Alzheimer's disease

Traumatic brain injury

Phobias

Chronic pain

Nocturnal myoclonus

Irritable bowel syndrome

Crohn's disease

Ulcerative colitis

Cognitive deterioration

Organ system dysfunction

Management of chronic stress

Cortisol dysfunction

\section{Appendix B}

Partial list of peripheral functions regulated by serotonin and/or dopamine

Regulation of phosphate

Loss of serotonin transporters associated with irritable

bowel syndrome

Hyperammonemia

Hyperammonemia associated with retardation

Regulation alterations in diabetes

Regulation of renal function

Regulation of renal hemodynamics

Blood pressure regulation

Potassium regulation

Sodium regulation

ATP regulation

Regulation of receptors outside the central nervous system including but not limited to:

- adrenal gland

- blood vessels

- carotid body

- intestines

- heart

- parathyroid gland

- kidney

- urinary tract

Regulation of renin secretion

Regulation by autocrine or paracrine fashion

Regulation in essential hypertension

Regulation of angiotensin II

Regulatory functions in shock

Regulatory functions in septic shock

Regulation of oxidative stress

Regulation of glomerular filtration

Regulation of functions that strengthen, examples include but are not limited to:

- bone marrow

- spleen

- lymph nodes

Regulation of dopamine in bone marrow cells including but not limited to:

- splenocytes

- lymphocytes from lymph nodes 
Regulation of sympathetic nervous system

Regulation of platelet function

Regulation of function in prostate cancer

Regulation of syncope due to carotid sinus hypersensitivity

Regulation of dialysis hypotension

Regulation of cardiophysiological function

Regulation of adrenochromaffin cells

Regulation in hypoxia-induced pulmonary hypertension

Regulation in Tourette's syndrome

Regulation of drug absorption and elimination

Regulation in pre-eclampsia

Regulation of fluid modulation and sodium intake via actions including but not limited to:

- central nervous system

- gastrointestinal tract

Regulation of tubular epithelial transport

Regulation of modulation of the secretion and/or action of vasopressin, which in turn causes changes in, but not limited to:

- renin

- aldosterone

- norepinephrine

- epinephrine

- endothelin B receptors

Regulation of fluid and sodium intake by way of "appetite" centers in the brain
Regulation in idiopathic hypertension

Regulation of alterations of gastrointestinal tract transport Regulation of detoxification of exogenous organic cations Regulation of prolactin secretion

Regulation affecting memory

Regulation of receptors in the central and peripheral system

Regulation of fluid and electrolyte balance including but not limited to:

- blood vessels

- gastrointestinal tract

- adrenal glands

- sympathetic nervous system

- hypothalamus

- other brain centers

Regulation of phosphorylation of DARPP-32

Regulation of dependent effects of psychostimulants and opioids

Regulation of neuronal differentiation

Regulation of neurotoxicity

Regulation of transcription

Regulatory effects on fibroblasts

Regulation of melatonin synthesis in photoreceptors

Cyclic regulation of intraocular pressure

\section{Publish your work in this journal}

The International Journal of General Medicine is an international, peer-reviewed open-access journal that focuses on general and internal medicine, pathogenesis, epidemiology, diagnosis, monitoring and treatment protocols. The journal is characterized by the rapid reporting of reviews, original research and clinical studies across all disease areas.
A key focus is the elucidation of disease processes and management protocols resulting in improved outcomes for the patient. The manuscript management system is completely online and includes a very quick and fair peer-review system. Visit http://www.dovepress.com/ testimonials.php to read real quotes from published authors. 